\title{
Echocardiographic assessment of right ventricular systolic function
}

\author{
Victor Chien-Chia $\mathrm{Wu}^{1,2}$, Masaaki Takeuchi ${ }^{1}$ \\ ${ }^{1}$ Department of Laboratory and Transfusion Medicine, University of Occupational and Environmental Health, School of Medicine, Kitakyushu, \\ Japan; ${ }^{2}$ Department of Cardiology, Chang Gung Memorial Hospital, Linkou Medical Center, Taoyuan \\ Contributions: (I) Concept and design: All authors; (II) Administrative support: M Takeuchi; (III) Provision of study materials or patients: M Takeuchi; \\ (IV) Collection and assembly of data: All authors; (V) Data analysis and interpretation: All authors; (VI) Manuscript writing: All authors; (VII) Final \\ approval of manuscript: All authors. \\ Correspondence to: Masaaki Takeuchi, MD, PhD, FASE, FESC, FJCC. Department of Laboratory and Transfusion Medicine, University of Occupational \\ and Environmental Health, School of Medicine, 1-1, Iseigaoka, Yahatanishi-ku, Kitakyushu 807-8555, Japan. Email: takeuchi@med.uoeh-u.ac.jp.
}

\begin{abstract}
Assessment of right chamber function has become increasingly popular and important in recent decades, complementing the evaluation of left chamber functional parameters. The late adoption and integration of right ventricular (RV) function into a complete evaluation of cardiac function could be due in part to the difficulty in visualization of the whole right ventricle, inconsistency in the analysis of RV parameters, and poor understanding of the impact of RV function on prognosis. From M-mode to 2-dimensional (2D) measurements of RV size and function, there have been arrays of parameters that provide important information of the right ventricle. However, there are limitations of using 2D echocardiography to quantify RV volume and ejection fraction (EF), and currently accurate measurements of RV volumes and EF can be obtained using cardiac magnetic resonance (CMR) imaging. Using 3-dimensional echocardiography (3DE), the measurements of RV volumes and EF have become feasible and reproducible. Importantly, 3D measurements have been validated against CMR with higher correlation compared to $2 \mathrm{D}$ measurements. With the establishment of reference values, 3D echocardiography determined RV volumes and EF have the potential for better understanding of RV function and exploration of its significance for outcome research in various clinical scenarios.
\end{abstract}

Keywords: Right ventricular function (RV function); 2-dimensional echocardiography (2DE); 3D echocardiography (3DE)

Submitted Jan 17, 2017. Accepted for publication Jun 02, 2017.

doi: $10.21037 / \mathrm{cdt} .2017 .06 .05$

View this article at: http://dx.doi.org/10.21037/cdt.2017.06.05

\section{Introduction}

Two-dimensional echocardiography (2DE) is an indispensable and integral part of clinical evaluation of patients with various cardiac diseases. However, most of the parameters derived from cardiac ultrasound examination mainly focus on the left chamber function, and less attention has been paid to the study and interpretation of right chamber function, namely the right ventricular (RV) systolic function. Early beliefs considered the importance of right ventricle is subservient to the left ventricle (1). This is because the knowledge of RV function lagged behind the left ventricular (LV) function and less is understood about the physiologic and prognostic roles of the right ventricle. Indeed, the inherent challenges in accurate assessment of irregular shaped RV cavity have hindered such progress. Strictly speaking, 2DE determined RV function parameters do not represent global function of the entire right ventricle, and it is almost impossible to precisely estimate RV volume and RV ejection fraction (RVEF) from few 2DE views. Nonetheless, right heart parameters are particularly important in patients whose diseases primarily affect the right ventricle, including congenital heart disease, pulmonary hypertension, valvular 
Table 1 Methods of echocardiographic evaluation of right ventricular function

\begin{tabular}{lcc}
\hline Modality & Unit & Abnormal \\
\hline M-mode echocardiography & $\mathrm{mm}$ & $<16$ \\
TAPSE & $\%$ & \\
2D echocardiography & $\%$ & $<35$ \\
RV FAC & & $<44$ \\
RV EF & $\mathrm{mmHg} / \mathrm{s}$ & $<400$ \\
Doppler echocardiography & & \\
dP/dt & - & $>0.40$ \\
RV MPI & - & $>0.55$ \\
Pulsed Doppler & $\mathrm{cm} / \mathrm{s}$ & $<10$ \\
Tissue Doppler & $\%$ & $>-20$ \\
RV S' & & $<44$ \\
Myocardial deformation echocardiography & \\
RV LS (free wall) & $\%$ & \\
3D echocardiography & $\%$ F & \\
RV EF & & \\
\hline
\end{tabular}

$\mathrm{EF}$, right ventricular ejection fraction; FAC, right ventricular fractional area change; LS, longitudinal strain; MPI, right ventricular myocardial performance index; TAPSE, tricuspid annular plane systolic excursion.

heart disease, ischemic heart disease, and chronic lung disease. RV function is also important in patients whose pathology is caused by the abnormality of the left heart chamber function. Recent advances in the study of RV function have made strides with establishment of reference values assessed by $3 \mathrm{D}$ echocardiography (3DE), from which cutoff values may provide predictive values for future events. In this review, we present a summary of RV systolic function parameters derived from 2DE and 3DE (Table 1) and their evidence and recommendations from current literature and guidelines on the acquisition, analysis, and application of these measurements.

\section{Anatomy of right ventricle}

The heart is located in the middle mediastinum, at the level of thoracic vertebrae T5-T8. The right ventricle is situated immediately posterior to the sternum and anteromedial to the left ventricle. The ratio of $\mathrm{RV}$ volume to $\mathrm{LV}$ volume is 0.91-1.27 with no gender differences (2), and the RV mass is approximately one-sixth of the LV mass (3). In contrast to the conical shape of the left ventricle, the shape of RV resembles a triangular crescentic shell attached to anterior and inferior left ventricle at interventricular septum, sharing the same border, and the right ventricle appears to wrap around part of the left ventricle. The right ventricle is divided into three major components, including the inflow tract, the trabeculated apex, and the infundibulum or RV outflow tract. Due to its arcuate nature, it has a shortaxis cross-sectional falcate shape. There are numerous trabeculae within the RV cavity with three prominent muscular bands: the parietal band, the septomarginal band, and the moderator band. The parietal band and the infundibular septum form the crista supraventricularis, and the septomarginal band extends inferiorly and connects with the moderator band to which it attaches to the anterior papillary muscle. To assess RV dimension, volume, and function, American Society of Echocardiography Guidelines suggest evaluation through apical 4-chamber, modified 4-chamber, left parasternal long-axis (PLAX) and parasternal short-axis (PSAX), left parasternal RV inflow, and subcostal views for comprehensive assessment of right ventricle (4).

\section{Physiology of right ventricle}

Right heart receives systemic venous return and pumps blood into pulmonary circulation, whereas left heart receives pulmonary venous return and pumps blood into systemic circulation. In theory, the right heart pumps about the same amount of blood as the left heart in each cardiac cycle. The right heart and left heart are connected in series and an interruption at any point of circulation can affect both right and left heart. Since right ventricle is filled with deoxygenated blood, RV wall is thinner than $\mathrm{LV}$ wall, and the chamber is highly compliant and very sensitive to preload. RV systolic function is a combination of preload, afterload, and contractility just like LV systolic function. Frank-Starling mechanism operates in the right ventricle where an increase in preload stretches RV wall resulting in enhanced myocardial contractility in the same way as left ventricle does. The RV preload is affected by intravascular volume status, ventricular compliance, ventricular relaxation, heart rate, and filling pressure. Under normal condition, the right heart pumps oxygendeprived blood with much lower pressure compared to the left heart, hence the right heart has lower impedance and higher distensibility. Isovolumic contraction time 

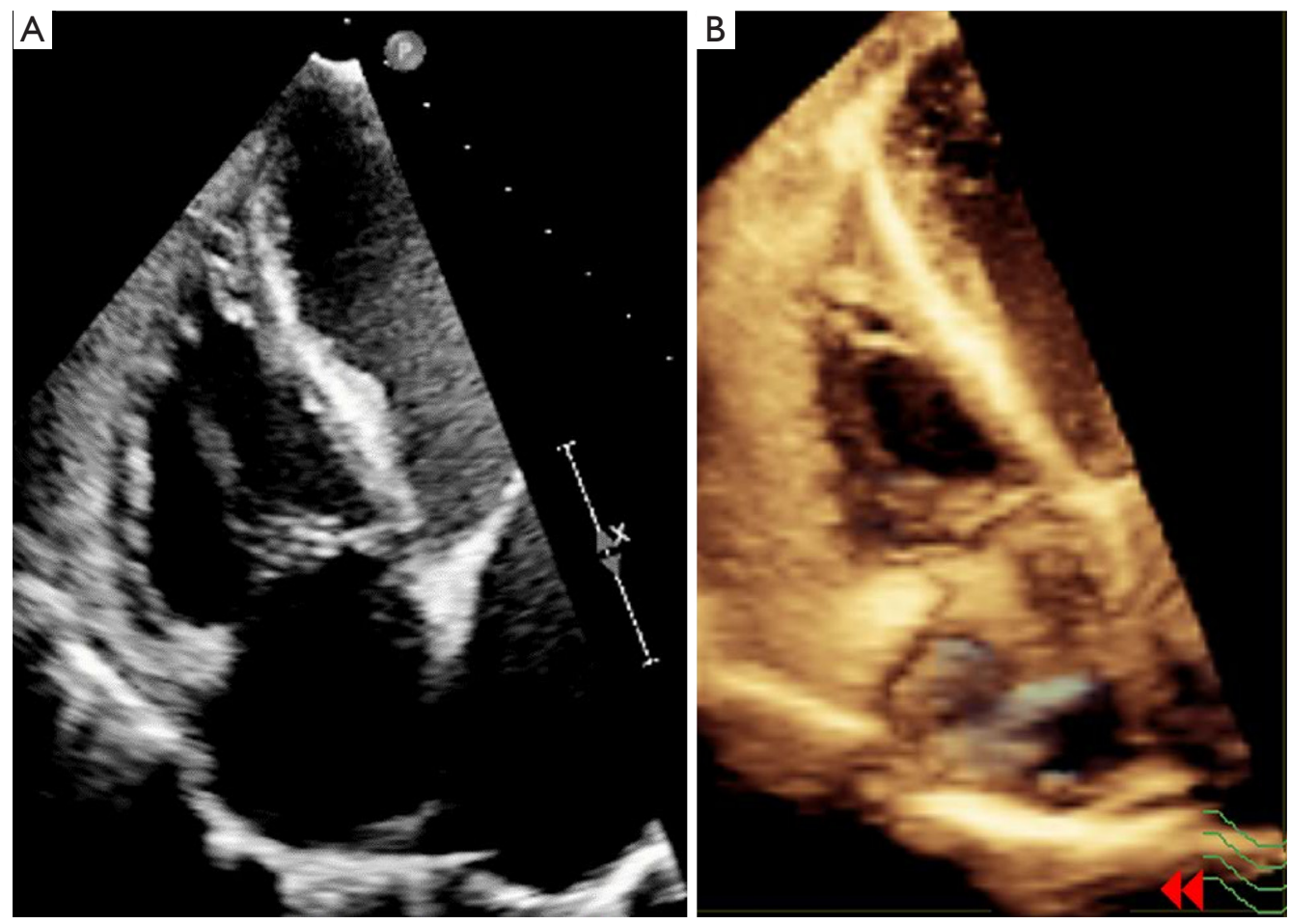

Figure 1 A representative case showing RV focused view on 2DE (A) and 3DE (B) in the same patient.

of the right ventricle is shorter than that of the left ventricle also due to $\mathrm{RV}$ pressure is able to rise faster to exceed the low pulmonary artery (PA) pressure (5). A study of RV pressure tracings show an early peaking and a rapidly declining pressure in contrast to the rounded contour of LV pressure tracing (6). The RV afterload is related to pulmonary vascular resistance (PVR) and PA pressure.

The understanding of complex relationship between RV contractility, preload, and afterload can be enhanced by the pressure-volume (P-V) loops. RV systolic pressure (RVSP) can be determined from peak tricuspid regurgitation (TR) velocity by the simplified Bernoulli equation in addition to right atrial (RA) pressure: $\mathrm{RVSP}=4(\mathrm{TR} \text { velocity })^{2}+$ RA pressure. PA systolic pressure is estimated also from TR velocity, and PA diastolic pressure is obtained by enddiastolic pulmonary regurgitation velocity. The normal mean PA pressure is less than $25 \mathrm{mmHg}$. In routine practice, RVSP is more commonly measured and normal resting value is defined as RVSP $\leq 35-36 \mathrm{mmHg}$ (peak TR velocity $\leq 2.8-2.9 \mathrm{~m} / \mathrm{s}$, assuming an RA pressure $3-5 \mathrm{mmHg}$ ) (4). Care must be taken to evaluate patients with increased PA pressure as afterload can be related to increased PVR. PVR can be estimated by the ratio of peak TR velocity over RV outflow tract velocity-time integral with units in Wood (7). A normal PVR is $<1.5$ Wood units and a significant pulmonary hypertension is defined as $>3$ Wood units (4).

In pathologic states that primarily affects the right ventricle, both volume and pressure overload can cause RV hypertrophy and enlargement. In diseases that causes $\mathrm{RV}$ pressure overload, RV wall thickens and in extreme cases such as congenital heart disease with Eisenmenger syndrome or severe pulmonary hypertension, the shape of ventricles is reversed, and the left ventricle becomes D-shaped. The complex interaction and relationship between RV contractility, preload, and afterload is better comprehended by P-V loop. The slop of this RV P-V loop is referred to as ventricular elastance, and is a reliable index of RV contractility (8).

\section{Importance of acquisition of the RV focused view}

Although the right ventricle can be visualized on the standard apical 4-chamber view, acquisition of RV-focused apical 4-chamber view is vital for its full delineation of the $\mathrm{RV}$ free wall, especially for the measurements of RV free wall longitudinal strain (LS) (Figure 1). The transducer 
should be placed more laterally to the point where the usual apical 4-chamber view is acquired. The sector size should be reduced as much as possible to increase frame rate. The same technique should also be applied when acquiring $3 \mathrm{DE}$ full volume datasets.

\section{Two-dimensional analysis of RV function}

The complex nature of RV contour makes it difficult to re-construct RV shape mentally utilizing even multiple $2 \mathrm{D}$ imaging planes. In addition, incorporation of these scanning planes still falls short of a complete $3 \mathrm{D}$ geometry, therefore echocardiographers have been using surrogate parameters to describe RV function. Among these, the most frequently reported $2 \mathrm{DE}$ measurements for $\mathrm{RV}$ function are tricuspid annular plane systolic excursion (TAPSE) and RV fractional area change (FAC) by the apical 4-chamber view. TAPSE is defined as the distance traveled between end-diastole and end-systole at the lateral corner of the tricuspid annulus. TAPSE has been validated to correlate strongly with RVEF measured by radionuclide angiography, with low observer variabilities (9). In addition, it has also been validated against RVEF by biplane Simpson method and RV FAC $(10,11)$. In a large series of 900 cases, including 150 normal subjects, a cutoff value $>17 \mathrm{~mm}$ of TAPSE is defined as normal and the guideline recommends the use of $<16 \mathrm{~mm}$ of TAPSE as abnormal $(4,12)$. Of note, TAPSE is angle dependent, and measurement can be affected by sliding motion of the heart within the chest cavity. TAPSE is also load dependent, and TAPSE is increased in the presence of severe TR while in the presence of mildly reduced RV systolic function, TAPSE may appear to be within normal range. TAPSE is a one-dimensional measurement which does not always reflect global RV function. However, it is an easily obtained surrogate marker for routine practice.

FAC is the area difference between RV end-diastolic and end-systolic areas measured through ideally RV-focused apical view. The border should be traced carefully to exclude the heavy trabeculations inside the right ventricle. FAC has been validated with cardiac magnetic resonance (CMR) (13) and shown to be an independent predictor for sudden death, mortality after pulmonary embolism, heart failure, stroke, and myocardial infarction (14-16). An FAC $<35 \%$ is considered abnormal by the guideline (4). FAC has the advantage of being quickly and easily obtainable parameter like TAPSE, and is one of the recommended methods alongside with TAPSE. However, since FAC is 2D measurement, its disadvantage is that it does not represent global RV function or actual RVEF.

2D RV volume quantification has been used with area-length methods and disk summation methods. The area-length method, based on the assumption of modified pyramidal or ellipsoid models for RV geometry approximation, underestimate CMR-derived RV volume and is inferior compared to $3 \mathrm{DE}$ derived volume $(17,18)$. The disk summation method, primarily adopted for calculating RV "body" volume, therefore underestimates true RV volume since RV inflow and outflow parts were not included during quantification. The pooled studies of these $2 \mathrm{DE}$ methods derived RVEF has lower reference value of $44 \%$ with $95 \%$ confidence interval (CI) $38-50 \%$ (4). However, calculation of 2D RVEF is currently not recommended by the American Society of Echocardiography.

\section{Doppler echocardiography analysis of RV function}

The rate of pressure rise in the ventricles $(\mathrm{dP} / \mathrm{dt})$ is validated as an index of ventricular contractility or systolic function. Initially described by Gleason and Braunwald in 1962 for both left and right ventricles (19), dP/dt of the right ventricle is calculated by measuring the time required for the TR jet to increase in velocity from 1 to $2 \mathrm{~m} / \mathrm{s}(20)$. Using the simplified Bernoulli formula, this is equivalent to a pressure rise from 4 to $16 \mathrm{mmHg}$ and a difference of $12 \mathrm{mmHg}$. Thus, $\mathrm{dP} / \mathrm{dt}$ is calculated by dividing $12 \mathrm{mmHg}$ to time in seconds. An $\mathrm{RV} \mathrm{dP/dt}<400 \mathrm{mmHg} / \mathrm{s}$ is likely abnormal. The advantage of the method is relative simple steps in the acquisition, measurement, and calculation. The disadvantage is that it is load dependent and is less accurate in patients with severe TR. Since there is lack of data in normal subjects, $\mathrm{RV} \mathrm{dP} / \mathrm{dt}$ cannot be recommended for its use in routine practice (4).

$\mathrm{RV}$ myocardial performance index (MPI), also known as Tei index, is a global parameter of both systolic and diastolic ventricular function (21). MPI is defined as the ratio of total isovolumic time divided by total ejection time (ET) by the formula IVRT + IVCT/ET. MPI has been shown to have good correlation with simultaneous recorded cardiac catheterization derived systolic peak $+\mathrm{dP} / \mathrm{dt}(\mathrm{r}=0.821$, $\mathrm{P}<0.001)$ and diastolic peak $-\mathrm{dP} / \mathrm{dt}(\mathrm{r}=0.833, \mathrm{P}<0.001)(22)$. MPI has shown to have predictive values in future events in patients with pulmonary hypertension, congenital heart disease, RV infarction, and hypertrophic cardiomyopathy (23-26). The upper reference limit for the RV MPI is 0.40 
using the pulsed Doppler method and 0.55 using the tissue Doppler method. The advantages include feasibility and reproducibility in wide range of subjects with or without TR. The disadvantages include that it is not used in patients with irregular heart beat and those whose RA pressure is elevated.

Tricuspid annular motion can be evaluated by tissue Doppler to measure the longitudinal velocity of the tricuspid annulus. This velocity has been named the RV S' or systolic excursion velocity. To measure this parameter, RV-focused view is used with tissue Doppler region of interest placed at the lateral corner of the tricuspid annulus acquired at high frame rate. The velocity $\mathrm{S}^{\prime}$ is read as the highest systolic velocity. A number of validation studies have been performed which showed good correlations to radionuclide angiography and CMR determined RVEF $(27,28)$. In fact, RV S' correlated better than FAC and TAPSE when RVEF by CMR was normal to mildly abnormal (defined as $<50 \%$ ) but second to FAC when RVEF by CMR was severely abnormal (defined as $<30 \%$ ) (29). A cutoff of RV S' $<11.5 \mathrm{~cm} / \mathrm{s}$ correlates with RVEF $<45 \%$ with a sensitivity of $90 \%$ and specificity of $85 \%$ and an RV $\mathrm{S}^{\prime}<10 \mathrm{~cm} / \mathrm{s}$ should raise the suspicion for abnormal RV function, particularly in younger adult patients (4).

\section{Myocardial deformation analysis of RV function}

Myocardial deformation, or strain, is defined as deformation of an object compared with its initial shape and is expressed as a percentage. RV LS is acquired using tissue Doppler imaging or speckle-tracking imaging. Since strain values by tissue Doppler imaging are angle dependent, RV LS is now measured by 2DE speckle tracking analysis. To calculate strain, high frame rates are required, thus narrow imaging sector focusing on the RV wall is desired. When analyzing RV LS, one can obtain RV free wall LS and RV global LS on the apical 4-chamber view. RV free wall LS is the strain in the RV free wall on the apical 4-chamber view. RV global LS is defined as averaged strain on the $R V$ free wall and interventricular septum. RV free wall strain is generally higher than RV global LS. Several studies have revealed its usefulness. RV free wall LS was evaluated in patients with systemic sclerosis with normal pulmonary pressure, and has been shown to detect early deterioration of RV function (30). Cutoff value of systolic LS at the basal free wall of $-25 \%$ yielded a $81 \%$ sensitivity and $82 \%$ specificity for the prediction of RVEF $>50 \%$ (31). In
80 patients with World Health Organization group 1 pulmonary hypertension, average RV free wall systolic strain worse than $-12.5 \%$ was associated with greater degree of disease progression within 6 months (32). A RV longitudinal peak systolic strain $\geq-19 \%$ was significant associated with all-cause mortality in 150 patients with pulmonary hypertension of different etiology (33). A larger study with 575 patients with pulmonary hypertension of various etiologies, RV strain predicted survival when adjusted for pulmonary pressure, PVR, and RA pressure (34). The advantages of RV free wall LS measurements include potential means to assess myocardial contractility that is less load dependent and can be applied in a wide variety of diseases. In a study of 276 healthy volunteers, normal values of RVLS were $-24.0 \pm 2.6 \%$ in men and $-26.7 \pm 3.1 \%$ for women (35). In a meta-analysis of 10 studies involving 486 patients without cardiopulmonary disease, the mean RV strain was $-26 \pm 4 \%$ (36). Currently, normal values of $\mathrm{RV}$ free wall strain were given as $-29.0 \pm 4.5 \%$ in the 2015 ASE/EACVI guidelines (37).

\section{Three-dimensional analysis of RV function}

At present, the determinations of $\mathrm{RV}$ volumes and $\mathrm{EF}$ are still provided by CMR. Limitations in the availability of the facility, portability, cost, and time have however largely confined its clinical use in selected number of subjects. $3 \mathrm{DE}$ holds good promise to $\mathrm{RV}$ volumetric analysis, without the need for geometric assumption. Although 3DE systematically underestimates RV volumes, several studies have shown $3 \mathrm{DE}$ determined RV volumes and function correlated well with CMR with steadily improvement seen over years (Table 2) (38-46). Using RV software in the earlier days, it took longer analysis time due to tedious manual editing of RV endocardial border in every patient. With improvement of image quality of $3 \mathrm{DE}$ and more user friendly RV quantification software, this drawback can be eliminated in the recent publications (46). Normal range of RV volumes and RVEF have been also established (Table 3) $(18,47,48)$. Recent publication has demonstrated the prognostic values of 3DE RVEF (49), and its clinical adoption in multicenter setting or certain cardiac diseases awaits further study. The advantages of 3DE are fast acquisition, independent of geometric assumptions, semi-automated border detection, dynamic RV cast visualization using dedicated software (Figures 2,3 and $S 1, S 2)$. The disadvantage of $3 \mathrm{DE}$ is similar to $2 \mathrm{DE}$ when imaging of $\mathrm{RV}$ free wall is not possible due to its proximity 


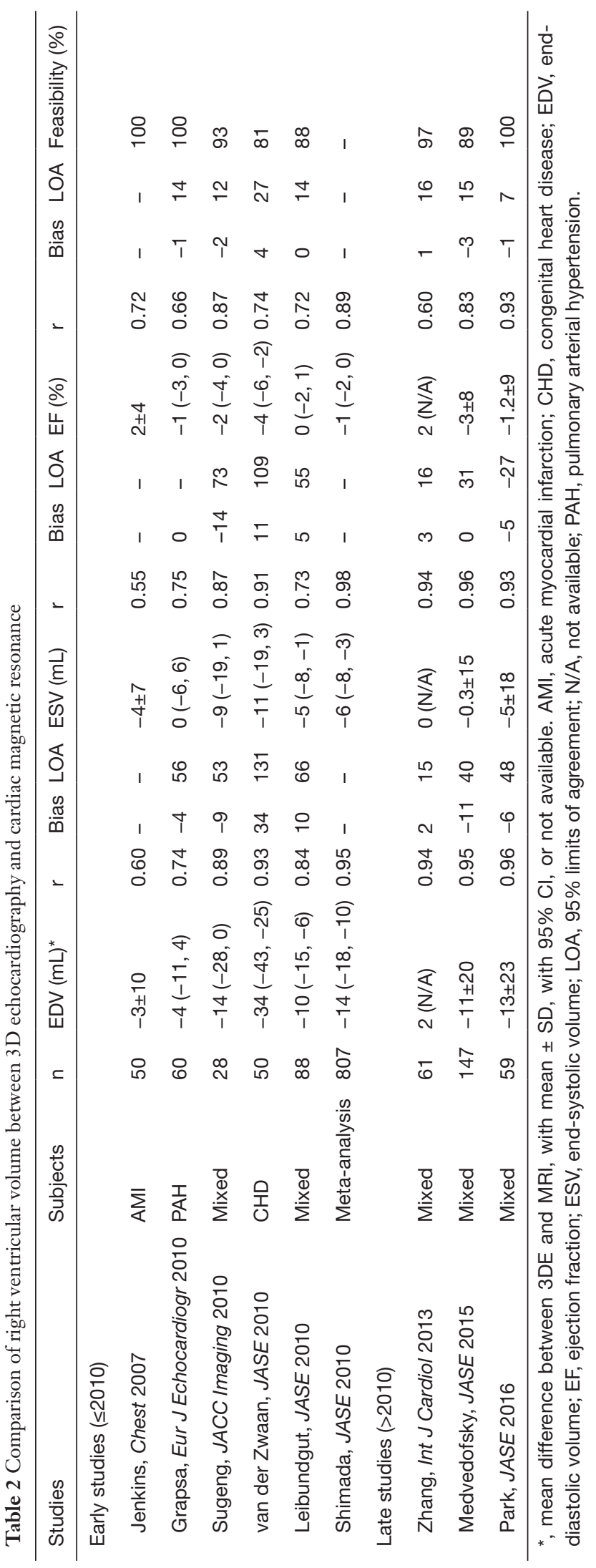

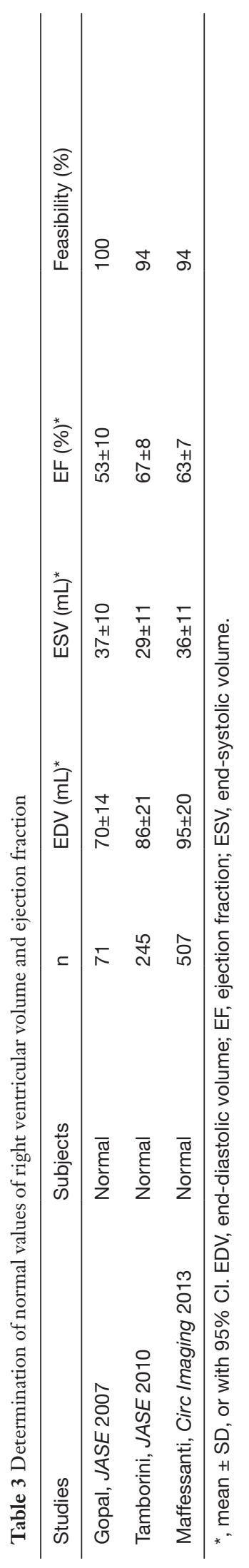




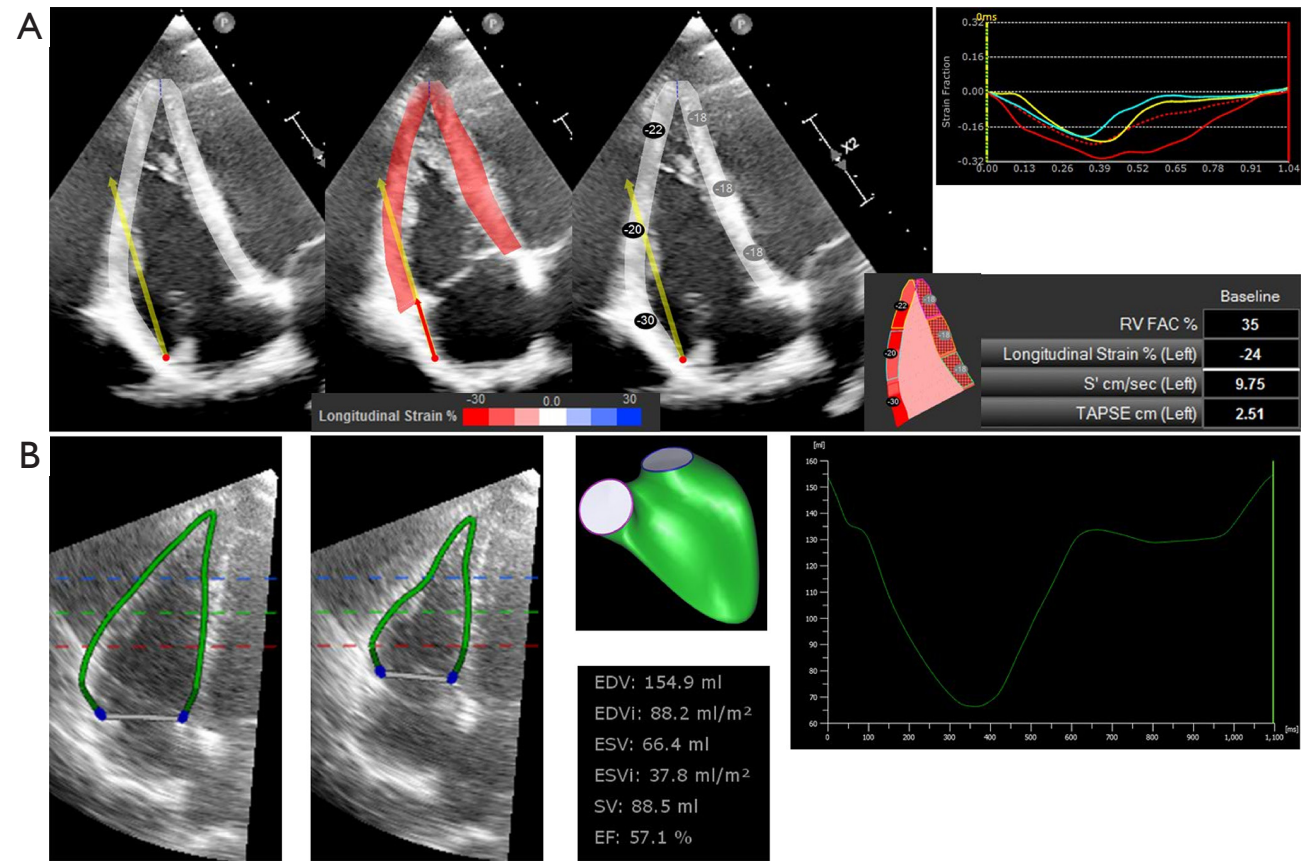

Figure 2 RV parameters measured by traditional 2DE (A), and by 3DE (B) in a patient with ischemic cardiomyopathy with normal RV systolic function. (A) 2D speckle tracking analysis of the right ventricle with vendor independent software (Echo Insight, Epsilon), which provides RV FAC, RV free wall strain, S' and TAPSE. In this particular case, all of these parameters are normal; (B) 3D speckle tracking analysis of the RV with vendor independent software (4D RV-Function 2, Tom Tec), which provides RV volumes and RVEF. RVEF is also normal. RV, right ventricular.

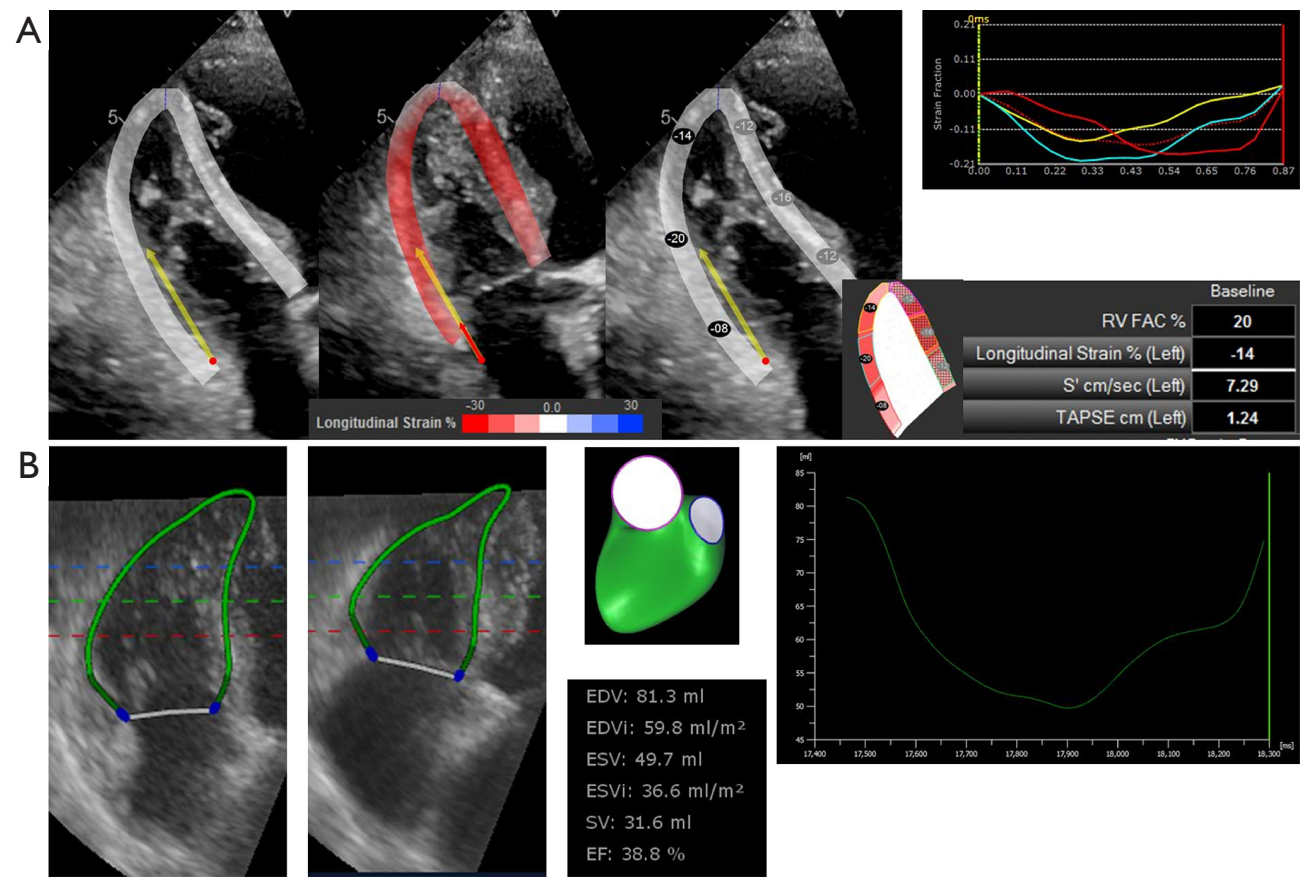

Figure $3 \mathrm{RV}$ parameters measured by traditional 2DE (A), and by 3DE (B) in a patient with pulmonary hypertension. In contrast to Figure 2, all 2D speckle tracking derived parameters were abnormal. 3D RVEF were also depressed to $38.8 \%$. 
to the sternum, a severely dilated RV cannot be imaged in its entirety by ultrasound probe.

\section{Conclusions}

Understanding the asymmetrical and complex RV shape is essential to accurate acquisition, analysis, and interpretation of currently proposed RV parameters. Evaluation of RV function by 2DE have been straightforward and also been validated against gold standard CMR and with prognostic values. Assessment of RV function by $3 \mathrm{DE}$ includes additional dimension for quantification of true RV volume and RVEF. Clinical application of $3 \mathrm{DE}$ determined parameters are expected to have enhanced outcome prediction in a variety of cardiac diseases.

\section{Acknowledgements}

None.

\section{Footnote}

Conflicts of Interest: The authors have no conflicts of interest to declare.

\section{References}

1. Haddad F, Hunt SA, Rosenthal DN, et al. Right ventricular function in cardiovascular disease, part I: Anatomy, physiology, aging, and functional assessment of the right ventricle. Circulation 2008;117:1436-48.

2. Altmayer SP, Patel AR, Addetia K, et al. Cardiac MRI right ventricle / left ventricle (RV/LV) volume ratio improves detection of RV enlargement. J Magn Reson Imaging 2016;43:1379-85.

3. Lorenz CH, Walker ES, Morgan VL, et al. Normal human right and left ventricular mass, systolic function, and gender differences by cine magnetic resonance imaging. J Cardiovasc Magn Reson 1999;1:7-21.

4. Rudski LG, Lai WW, Afilalo J, et al. Guidelines for the echocardiographic assessment of the right heart in adults: a report from the American Society of Echocardiography endorsed by the European Association of Echocardiography, a registered branch of the European Society of Cardiology, and the Canadian Society of Echocardiography. J Am Soc Echocardiogr 2010;23:685713; quiz 786-8.

5. Haddad F, Doyle R, Murphy DJ, et al. Right ventricular function in cardiovascular disease, part II: pathophysiology, clinical importance, and management of right ventricular failure. Circulation 2008;117:1717-31.

6. Dell'Italia LJ, Walsh RA. Acute determinants of the hangout interval in the pulmonary circulation. Am Heart J 1988;116:1289-97.

7. Abbas AE, Fortuin FD, Schiller NB, et al. A simple method for noninvasive estimation of pulmonary vascular resistance. J Am Coll Cardiol 2003;41:1021-7.

8. Starling MR, Walsh RA, Dell'Italia LJ, et al. The relationship of various measures of end-systole to left ventricular maximum time-varying elastance in man. Circulation 1987;76:32-43.

9. Kaul S, Tei C, Hopkins JM, et al. Assessment of right ventricular function using two-dimensional echocardiography. Am Heart J 1984;107:526-31.

10. López-Candales A, Dohi K, Rajagopalan N, et al. Defining normal variables of right ventricular size and function in pulmonary hypertension: an echocardiographic study. Postgrad Med J 2008;84:40-5.

11. Miller D, Farah MG, Liner A, et al. The relation between quantitative right ventricular ejection fraction and indices of tricuspid annular motion and myocardial performance. J Am Soc Echocardiogr 2004;17:443-7.

12. Tamborini G, Pepi M, Galli CA, et al. Feasibility and accuracy of a routine echocardiographic assessment of right ventricular function. Int J Cardiol 2007;115:86-9.

13. Anavekar NS, Gerson D, Skali H, et al. Twodimensional assessment of right ventricular function: an echocardiographic-MRI correlative study. Echocardiography 2007;24:452-6.

14. Nass N, McConnell MV, Goldhaber SZ, et al. Recovery of regional right ventricular function after thrombolysis for pulmonary embolism. Am J Cardiol 1999;83:804-6, A10.

15. Zornoff LA, Skali H, Pfeffer MA, et al. Right ventricular dysfunction and risk of heart failure and mortality after myocardial infarction. J Am Coll Cardiol 2002;39:1450-5.

16. Anavekar NS, Skali H, Bourgoun M, et al. Usefulness of right ventricular fractional area change to predict death, heart failure, and stroke following myocardial infarction (from the VALIANT ECHO Study). Am J Cardiol 2008;101:607-12.

17. Helbing WA, Bosch HG, Maliepaard C, et al. Comparison of echocardiographic methods with magnetic resonance imaging for assessment of right ventricular function in children. Am J Cardiol 1995;76:589-94.

18. Gopal AS, Chukwu EO, Iwuchukwu CJ, et al. Normal values of right ventricular size and function by real-time 
3-dimensional echocardiography: comparison with cardiac magnetic resonance imaging. J Am Soc Echocardiogr 2007;20:445-55.

19. Gleason WL, Braunwald E. Studies on the first derivative of the ventricular pressure pulse in man. J Clin Invest 1962;41:80-91.

20. Anconina J, Danchin N, Selton-Suty C, et al. Noninvasive estimation of right ventricular $\mathrm{dP} / \mathrm{dt}$ in patients with tricuspid valve regurgitation. Am J Cardiol 1993;71:1495-7.

21. Tei C, Dujardin KS, Hodge DO, et al. Doppler echocardiographic index for assessment of global right ventricular function. J Am Soc Echocardiogr 1996;9:838-47.

22. Tei C, Nishimura RA, Seward JB, et al. Noninvasive Doppler-derived myocardial performance index: correlation with simultaneous measurements of cardiac catheterization measurements. J Am Soc Echocardiogr 1997;10:169-78.

23. Sebbag I, Rudski LG, Therrien J, et al. Effect of chronic infusion of epoprostenol on echocardiographic right ventricular myocardial performance index and its relation to clinical outcome in patients with primary pulmonary hypertension. Am J Cardiol 2001;88:1060-3.

24. Eidem BW, O'Leary PW, Tei C, et al. Usefulness of the myocardial performance index for assessing right ventricular function in congenital heart disease. Am J Cardiol 2000;86:654-8.

25. Møller JE, Søndergaard E, Poulsen SH, et al. Serial Doppler echocardiographic assessment of left and right ventricular performance after a first myocardial infarction. J Am Soc Echocardiogr 2001;14:249-55.

26. Mörner S, Lindquist P, Waldenström A, et al. Right ventricular dysfunction in hypertrophic cardiomyopathy as evidenced by the myocardial performance index. Int $\mathrm{J}$ Cardiol 2008;124:57-63.

27. Schwerzmann M, Samman AM, Salehian O, et al. Comparison of echocardiographic and cardiac magnetic resonance imaging for assessing right ventricular function in adults with repaired tetralogy of fallot. Am J Cardiol 2007;99:1593-7.

28. Ueti OM, Camargo EE, Ueti Ade A, et al. Assessment of right ventricular function with Doppler echocardiographic indices derived from tricuspid annular motion: comparison with radionuclide angiography. Heart 2002;88:244-8.

29. Pavlicek M, Wahl A, Rutz T, et al. Right ventricular systolic function assessment: rank of echocardiographic methods vs. cardiac magnetic resonance imaging. Eur J Echocardiogr 2011;12:871-80.

30. Borges AC, Knebel F, Eddicks S, et al. Right ventricular function assessed by two-dimensional strain and tissue Doppler echocardiography in patients with pulmonary arterial hypertension and effect of vasodilator therapy. Am J Cardiol 2006;98:530-4.

31. Vitarelli A, Conde Y, Cimino E, et al. Assessment of right ventricular function by strain rate imaging in chronic obstructive pulmonary disease. Eur Respir J 2006;27:268-75.

32. Sachdev A, Villarraga HR, Frantz RP, et al. Right ventricular strain for prediction of survival in patients with pulmonary arterial hypertension. Chest 2011;139:1299-309.

33. Haeck ML, Scherptong RW, Marsan NA, et al. Prognostic value of right ventricular longitudinal peak systolic strain in patients with pulmonary hypertension. Circ Cardiovasc Imaging 2012;5:628-36.

34. Fine NM, Chen L, Bastiansen PM, et al. Outcome prediction by quantitative right ventricular function assessment in 575 subjects evaluated for pulmonary hypertension. Circ Cardiovasc Imaging 2013;6:711-21.

35. Muraru D, Onciul S, Peluso D, et al. Sex- and MethodSpecific Reference Values for Right Ventricular Strain by 2-Dimensional Speckle-Tracking Echocardiography. Circ Cardiovasc Imaging 2016;9:e003866.

36. Fine NM, Chen L, Bastiansen PM, et al. Reference Values for Right Ventricular Strain in Patients without Cardiopulmonary Disease: A Prospective Evaluation and Meta-Analysis. Echocardiography 2015;32:787-96.

37. Lang RM, Badano LP, Mor-Avi V, et al. Recommendations for cardiac chamber quantification by echocardiography in adults: an update from the American Society of Echocardiography and the European Association of Cardiovascular Imaging. J Am Soc Echocardiogr 2015;28:1-39.e14.

38. Jenkins C, Chan J, Bricknell K, et al. Reproducibility of right ventricular volumes and ejection fraction using realtime three-dimensional echocardiography: comparison with cardiac MRI. Chest 2007;131:1844-51.

39. Grapsa J, O'Regan DP, Pavlopoulos H, et al. Right ventricular remodelling in pulmonary arterial hypertension with three-dimensional echocardiography: comparison with cardiac magnetic resonance imaging. Eur J Echocardiogr 2010;11:64-73.

40. Sugeng L, Mor-Avi V, Weinert L, et al. Multimodality comparison of quantitative volumetric analysis of the right ventricle. JACC Cardiovasc Imaging 2010;3:10-8.

41. van der Zwaan HB, Helbing WA, McGhie JS, et al. Clinical value of real-time three-dimensional echocardiography for right ventricular quantification in congenital heart disease: 
validation with cardiac magnetic resonance imaging. J Am Soc Echocardiogr 2010;23:134-40.

42. Leibundgut G, Rohner A, Grize L, et al. Dynamic assessment of right ventricular volumes and function by real-time three-dimensional echocardiography: a comparison study with magnetic resonance imaging in 100 adult patients. J Am Soc Echocardiogr 2010;23:116-26.

43. Zhang QB, Sun JP, Gao RF, et al. Feasibility of singlebeat full-volume capture real-time three-dimensional echocardiography for quantification of right ventricular volume: validation by cardiac magnetic resonance imaging. Int J Cardiol 2013;168:3991-5.

44. Medvedofsky D, Addetia K, Patel AR, et al. Novel Approach to Three-Dimensional Echocardiographic Quantification of Right Ventricular Volumes and Function from Focused Views. J Am Soc Echocardiogr 2015;28:1222-31.

45. Park JB, Lee SP, Lee JH, et al. Quantification of Right Ventricular Volume and Function Using Single-Beat Three-Dimensional Echocardiography: A Validation Study with Cardiac Magnetic Resonance. J Am Soc Echocardiogr 2016;29:392-401.

Cite this article as: $\mathrm{Wu} \mathrm{VC}$, Takeuchi $\mathrm{M}$. Echocardiographic assessment of right ventricular systolic function. Cardiovasc Diagn Ther 2018;8(1):70-79. doi: 10.21037/cdt.2017.06.05
46. Muraru D, Spadotto V, Cecchetto A, et al. New speckletracking algorithm for right ventricular volume analysis from three-dimensional echocardiographic data sets: validation with cardiac magnetic resonance and comparison with the previous analysis tool. Eur Heart J Cardiovasc Imaging 2016;17:1279-89.

47. Tamborini G, Marsan NA, Gripari P, et al. Reference values for right ventricular volumes and ejection fraction with real-time three-dimensional echocardiography: evaluation in a large series of normal subjects. J Am Soc Echocardiogr 2010;23:109-15.

48. Maffessanti F, Muraru D, Esposito R, et al. Age-, body size-, and sex-specific reference values for right ventricular volumes and ejection fraction by three-dimensional echocardiography: a multicenter echocardiographic study in 507 healthy volunteers. Circ Cardiovasc Imaging 2013;6:700-10.

49. Nagata Y, Wu VC, Kado Y, et al. Prognostic Value of Right Ventricular Ejection Fraction Assessed by Transthoracic 3D Echocardiography. Circ Cardiovasc Imaging 2017;10. pii: e005384. 


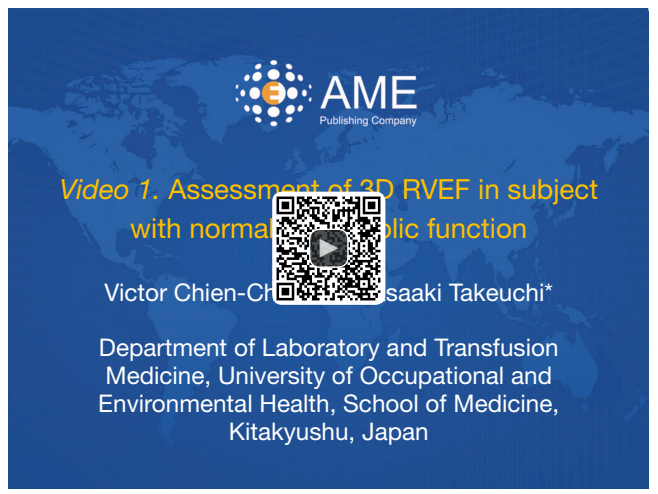

Figure S1 Assessment of 3D RVEF in subject with normal RV systolic function (50). RVEF, right ventricular ejection fraction. Available online: http://asvidett.amegroups.com/article/view/22723

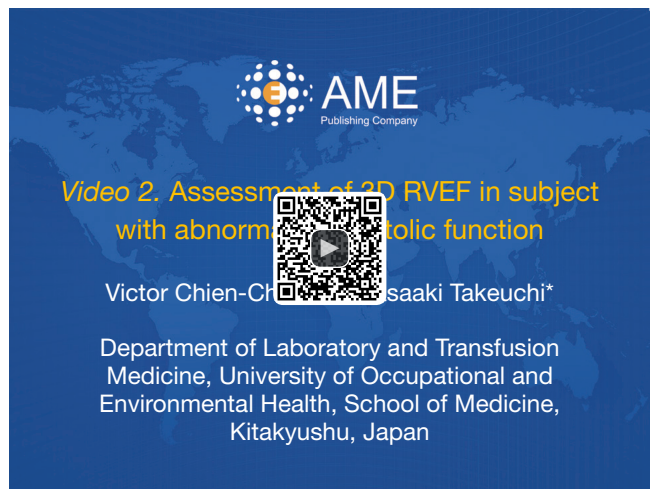

Figure S2 Assessment of 3D RVEF in subject with abnormal RV systolic function (51). RVEF, right ventricular ejection fraction.

Available online: http://asvidett.amegroups.com/article/view/22724

\section{References}

50. Wu VC, Takeuchi M. Assessment of 3D RVEF in subject with normal RV systolic function. Asvide 2018;5:062. Available online: http://asvidett.amegroups.com/article/view/22723

51. Wu VC, Takeuchi M. Assessment of 3D RVEF in subject with abnormal RV systolic function. Asvide 2018;5:063. Available online: http://asvidett.amegroups.com/article/view/22724 